\title{
TMS-induced neural noise in sensory cortex interferes with short-term memory storage in prefrontal cortex
}

\section{Tyler D. Bancroft *, Jeremy Hogeveen, William E. Hockley and Philip Servos}

Department of Psychology, Wilfrid Laurier University, Waterloo, ON, Canada

\section{Edited by:}

Nicolas Brunel, Centre National de la Recherche Scientifique, France

Reviewed by:

Da-Hui Wang, Beijing Normal

University, China

Gianluigi Mongillo, Paris Descartes

University, France

*Correspondence:

Tyler D. Bancroft, Department of

Psychology, Wilfrid Laurier

University, 75 University Avenue

West, Waterloo, ON N2L 3C5,

Canada

e-mail: banc6110@mylaurier.ca
In a previous study, Harris et al. (2002) found disruption of vibrotactile short-term memory after applying single-pulse transcranial magnetic stimulation (TMS) to primary somatosensory cortex (SI) early in the maintenance period, and suggested that this demonstrated a role for $\mathrm{SI}$ in vibrotactile memory storage. While such a role is compatible with recent suggestions that sensory cortex is the storage substrate for working memory, it stands in contrast to a relatively large body of evidence from human EEG and single-cell recording in primates that instead points to prefrontal cortex as the storage substrate for vibrotactile memory. In the present study, we use computational methods to demonstrate how Harris et al.'s results can be reproduced by TMS-induced activity in sensory cortex and subsequent feedforward interference with memory traces stored in prefrontal cortex, thereby reconciling discordant findings in the tactile memory literature.

Keywords: short-term memory, working memory, scalar memory, TMS, vibrotactile, noise, computational modeling

\section{INTRODUCTION}

Vibrotactile short-term memory [often referred to as vibrotactile working memory (VWM)] is a powerful paradigm for studying the behavioral and neural correlates of working and short-term memory (Bancroft et al., 2011a). VWM tasks usually involve presenting subjects with two vibrational stimuli delivered to the hand (the target and the probe), separated by an unfilled delay period, and instruct subjects to report whether the two stimuli are of same or different frequencies, or whether the probe is of a higher or lower frequency than the target. Notably, the salient stimulus feature (vibrational frequency) can be represented as a scalar value, and the firing rates of neurons encoding vibrotactile stimuli tend to be monotonic functions of stimulus frequency (Romo et al., 1999; Romo and Salinas, 2003). This makes vibrotactile memory a useful paradigm for integrating research results across various research methodologies, and recent studies have taken advantage of this property by demonstrating that it is possible to decode the stimulus frequency held in memory from beta-band EEG activity in frontal cortex (Spitzer et al., 2010, 2014; Spitzer and Blankenburg, 2011, 2012). Intriguingly, recent research has suggested that vibrotactile memory may be one of a family of scalar short-term memory tasks, including auditory memory for pure tones and memory for the frequency of visual flicker (Spitzer and Blankenburg, 2012), as well as stimulus amplitude and duration (Spitzer et al., 2014), that appear to share a similar, supramodal neural code in both sensory cortex and higher cortical regions, and rely on the same region of prefrontal cortex as a storage substrate.

An intriguing study, however, poses a challenge to this interpretation of results. Harris et al. (2002) presented subjects with two vibrotactile stimuli, separated by an unfilled delay period, and asked them to compare the stimuli. During the delay period, they applied single-pulse transcranial magnetic stimulation (TMS) to primary somatosensory cortex (SI). This study employed a "virtual lesion" design, in which TMS-induced changes in behavior suggest a causal relationship between peri-stimulation neural activity and task-related perceptual and cognitive functions (Robertson et al., 2003). Harris et al. (2002) found a significant decrease in performance when the TMS pulse was applied to contralateral SI (relative to ipsilateral SI) 300 or $600 \mathrm{~ms}$ into a $1500 \mathrm{~ms}$ delay period, but not when it was applied 900 or $1200 \mathrm{~ms}$ into the delay period. (Note that while the decrease in performance in response to the $900 \mathrm{~ms}$ onset TMS pulse did not reach statistical significance ( $p=0.16)$, a trend is visible.) In contrast, TMS to ipsilateral SI did not significantly reduce performance. Harris et al. suggested that contralateral SI acts as a memory storage system for VWM. Such a notion is consistent with a previous single-cell recording study that reports SI encoding of complex tactile stimuli (Zhou and Fuster, 1996).

However, this notion conflicts with recent findings from human EEG studies and single-cell recording in non-human primates. Various studies by the research group of Romo et al. have suggested that regions in prefrontal cortex are the storage substrate used during VWM tasks and that no representation of the stored stimulus persists across the delay period in SI (see Romo and Salinas, 2003, for a review), and recent EEG studies by Spitzer and colleagues have reported being able to decode the frequency of a stored vibrational stimulus from prefrontal beta-band activity during the delay period of VWM (and other scalar STM) tasks (Spitzer et al., 2010, 2014; Spitzer and Blankenburg, 2011, 2012). The apparent incompatibility of these findings and those of Harris et al. (2002) raises questions about the scalar memory interpretation of results from VWM research, and also about whether the neural systems underlying VWM differ between humans and non-human primates.

The location of VWM storage has important implications for working and short-term memory theory, and the factors that determine storage location are unresolved. Postle (2006) 
suggested that stimuli tend to be stored in relevant regions of cortex that have pre-existing representations of that type of stimulus, such as sensory cortex; in order to account for recent experimental findings (including those around vibrotactile memory), we have recently suggested that less complex stimuli with simple neural codes instead tend to be stored in prefrontal cortex (Bancroft et al., 2014). As this theoretical framework is partly based on research showing prefrontal storage of scalar stimuli, reconciling Harris et al.'s (2002) results with other findings (i.e., Romo and Salinas, 2003; Spitzer et al., 2010) has theoretical importance.

We offer an alternative interpretation of Harris et al.'s (2002) findings. According to the former view, the application of TMS suppressed neural activity within SI during the delay period, and the consequent impact on VWM performance can be interpreted as evidence that SI is involved in VWM storage. However, beyond local changes in cortical activity, TMS can induce distal effects at brain regions receiving feedforward inputs from the targeted brain region (e.g., Paus et al., 1997). Rather than SI being a storage medium for vibrotactile memory, we suggest that the application of TMS induces or increases activity in sensory cortex (both in SI and in secondary somatosensory cortex (SII), via feedforward connections), and that this activity then interferes with VWM storage in PFC.

It has been established that TMS can induce neural activity when applied to some areas of sensory cortex, including somatosensory cortex (Sugishita and Takayama, 1993; Ray et al., 1998; Stewart et al., 2001; Ptito et al., 2008). As well, recent behavioral and computational studies have suggested that when irrelevant vibrotactile stimuli are presented during the maintenance period of a VWM task, they reduce performance by being encoded into memory (Bancroft and Servos, 2011; Bancroft et al., 2011b, 2012, 2013). As there is a direct mapping between induced activity in SI and the frequency of the stimulus perception created by that induced activity (e.g., Romo et al., 1998), it follows that increased activity in SI due to TMS could have similar effects to irrelevant somatosensory stimuli.

Perhaps most compellingly, somatosensory memory studies that have used TMS to increase activity in the middle frontal gyrus (a region of prefrontal cortex known to inhibit activity in SI) have reported decreased response times when TMS was applied early (300 ms onset) but not late (1200 ms onset) in the delay period, suggesting a decrease in interference (Hannula et al., 2010; also see Savolainen et al., 2011). Given that these TMS manipulations, known to suppress activity in SI, have been shown to improve, not reduce, performance on tactile memory tasks, it raises an interesting question: Is Harris et al.'s (2002) manipulation suppressing activity in SI, or is it producing excitatory or facilitatory effects that impact storage systems further downstream?

In the present study, we adapted a computational model of prefrontal cortex (Miller and Wang, 2006) in order to demonstrate that Harris et al.'s (2002) results can be produced by TMS-induced activity in sensory cortex, resulting in interference with information stored in prefrontal cortex. As pointed out by Miller and Wang, feeding noise into an integrator causes a decrease in performance proportional to the duration of noise. In the present study, the accumulation of noise in PFC leads to an inverse relationship between task performance and the delay between TMS offset and probe onset.

\section{MODEL}

The model used in the present study was originally developed by Miller and Wang (2006) as a model of prefrontal neurons involved in VWM tasks. We have previously adapted it to model the interfering effects of distractor stimuli on VWM (Bancroft et al., 2013). It is a rate model, based on the interaction of pairs of populations of prefrontal neurons. While the Miller and Wang model operates at a relatively high level of abstraction, it captures the fundamentally subtractive nature of the stimulus comparison process (Romo and Salinas, 2003), and has proven capable of fitting a variety of experimental data (e.g., Bancroft et al., 2013). In addition, the model can be fit to data with relatively few free parameters, which is beneficial when fitting a dataset with relatively few data points (such as the Harris et al. data we consider in this paper).

In this model, comparison $(C)$ populations receive input from sensory cortex and have excitatory outputs to populations of memory $(M)$ neurons. Memory populations have excitatory self-connections (allowing persistent activity in the absence of external input), and inhibitory connections to $C$ populations. The equations governing the behavior of the network are as follows:

$$
\begin{gathered}
d r_{C} / d t=(1 / \tau)\left(-r_{C}+w_{M C} r_{M}+w_{I C} I\right) \\
d r_{M} / d t=(1 / \tau)\left(-r_{M}+w_{M M} r_{M}+w_{C M} r_{C}\right)
\end{gathered}
$$

where $r$ is the firing rate of a population, $\tau$ is a time constant, $w_{A B}$ represents the strength of a connection from a population $A$ to another population $B$, and $I$ is the input received from sensory cortex. The addition of $w_{I C}$ to the model is intended as a potential scaling factor to allow presentation of stimulus frequencies outside of biologically-realistic firing rates (for example, auditory stimuli in the $\mathrm{kHz}$ range).

Note that if $w_{M M}$ is set to 1 , the $M$ population becomes a perfect (i.e., lossless) integrator, and the equation governing behavior of $M$ populations can be reduced to:

$$
d r_{M} / d t=(1 / \tau)\left(w_{C M} r_{C}\right)
$$

We have used this reduced equation in the present study. Upon presentation of a target stimulus, a $C$ population transmits the stimulus frequency to an $M$ population. The $M$ population then inhibits activity in the $C$ population, driving the $C$ firing rate back to baseline. The self-connection allows the $M$ population to maintain its firing rate in the absence of external stimulation. Upon presentation of the probe stimulus to the $C$ population, the combination of inhibitory input from the $M$ population and excitatory input from sensory cortex results in the $C$ population calculating some function of $f_{\text {target }}-f_{\text {probe }}$, consistent with experimental findings (Romo and Salinas, 2003), and also consistent with decision-making mechanisms used in abstract mathematical models of VWM (Bancroft et al., 2012). Note that experimental findings have reported finding neurons in sensory cortex that 
have firing rates that are positive monotonic functions of stimulus frequency, as well as neurons that have negative monotonic functions of stimulus frequency (Romo and Salinas, 2003). This plays an important role in the functioning of the model. $C$ populations that receive positive monotonic input (we refer to these as $C_{+}$populations) will fire above baseline when the probe stimulus is a higher frequency than the target stimulus, while populations that receive negative monotonic input ( $C_{-}$populations) will act as detectors for lower-frequency probes.

We have also added decision $(D)$ populations to the model to facilitate decision-making. The $D$ populations receive excitatory output from $C$ populations during the presentation of probe stimuli:

$$
d r_{D} / d t=(1 / \tau)\left(w_{C D} r_{C}\right)
$$

During target presentation and the delay period, $w_{C D}$ is set to 0 , and only assumes a non-zero value upon presentation of the probe stimulus. During probe presentation, the $D$ populations act as perfect integrators of the activity of the relevant $C$ population; this allows a direct comparison between the total activities of the $C_{+}$and $C_{-}$populations (and therefore the probe-higher and probe-lower detectors).

In the present study, we simulated two triplets of $C / M / D$ populations (see Figure 1), one receiving positive monotonic input (with subscript + ), the other receiving negative monotonic input (with subscript - ). The triplets were not connected to each other. To determine a simulated response, we compared the activity of the $D_{+}$and $D_{-}$populations shortly after probe offset. If activity in the $D_{+}$population exceeded that in the $D_{-}$population,

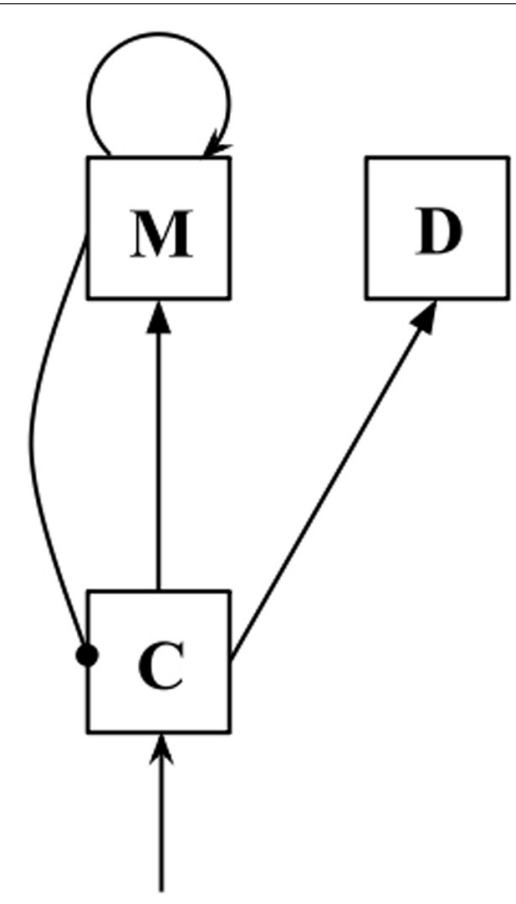

FIGURE 1 | Diagram of a C/M/D triplet. Arrows indicate excitatory connections, while lines ending in circles indicate inhibitory connections. it follows that overall activity in the $C_{+}$population exceeded that in the $C_{-}$population across the probe presentation period, and we recorded a probe-higher response. If activity in the $D_{-}$ population exceeded that of the $D_{+}$population, we recorded a probe-lower response, and if activity in the two populations was equal, a response was randomly chosen.

During the delay period, the model received constant input, with input values drawn from an exponential distribution with the distribution parameter $\lambda$, inversely proportional to the mean and variance of the distribution. This noisy input represents ongoing, baseline activity in sensory regions. Critically, we modeled the application of TMS to sensory cortex by allowing $\lambda$ to vary as a free parameter. If TMS increases activity in sensory cortex, we would expect the magnitude of the noise to increase (and therefore the value of $\lambda$ to decrease). Further, allowing values of $\lambda$ to vary separately for ipsilateral and contralateral stimulation allows us to test for differing effects of inhibition depending on laterality-if ipsilateral SI is more greatly inhibited than contralateral SI, we would expect a smaller magnitude of interference (and therefore a greater value for $\lambda$ ). The exponential distribution was chosen for this study as it has one parameter that determines both the mean and the variance of the distribution.

\section{SIMULATION METHODOLOGY}

In the present study, input to PFC was of two types. During target and probe presentation, $C_{+}$populations received input equal to $w_{I C} f$, and $C_{-}$populations received input equal to $w_{I C}(40-f)$, where $f$ was the frequency (in $\mathrm{Hz}$ ) of the stimulus, and $w_{I C}$ was the strength of the connection from sensory cortex to prefrontal cortex. Consistent with previous work (Bancroft et al., 2013), stimulus frequency $(f)$ was drawn from a Gaussian distribution with a mean equal to that of the presented stimulus, and standard deviation $(\sigma)$ allowed to vary as a free parameter, in order to account for inaccuracy in the neural signal introduced during neural transmission and processing. Firing rates $\left(r_{\text {population }}\right)$ were not allowed to decrease below zero. Other parameter values are presented in Table 1.

During the delay period, $C$ populations received noisy input drawn from an exponential distribution at each integration timestep, with the distribution parameter $\lambda$ set as a free

\section{Table 1 | Simulation parameters.}

\begin{tabular}{ll}
\hline Parameter & Value \\
\hline$f_{\text {target }}$ & $20 \mathrm{~Hz}$ \\
$f_{\text {probe, higher }}$ & $22 \mathrm{~Hz}$ \\
$f_{\text {probe, lower }}$ & $18 \mathrm{~Hz}$ \\
Stimulus duration & $1000 \mathrm{~ms}$ \\
Delay period duration & $1500 \mathrm{~ms}$ \\
$\tau$ & 10 \\
$W_{I C}$ & 0.4 \\
$W_{C M}$ & 0.4 \\
$w_{M C}$ & -0.4 \\
$w_{C D}$ & 0 (during target presentation/delay \\
& periods); 0.5 (during probe presentation) \\
$r_{\text {minimum }}$ & 0
\end{tabular}


parameter. The parameter $\lambda$ determines the mean $(1 / \lambda)$ and variance $\left(1 / \lambda^{2}\right)$ of an exponential distribution.

Harris et al. (2002 Exp. 2) presented subjects with two $1000 \mathrm{~ms}$ vibrotactile stimuli (the target and probe), separated by a $1500 \mathrm{~ms}$ delay period. TMS was applied to either ipsilateral or contralateral SI, at an onset of either $300,600,900$, or $1200 \mathrm{~ms}$ into the delay period. The target and probe stimuli differed by $\pm 2 \mathrm{~Hz}$, and subjects were required to report whether the probe was of a higher or lower frequency than the target.

To simulate the effects of TMS, $\lambda$ was allowed to assume two values during the delay period: The initial value $\left(\lambda_{\text {baseline }}\right)$, and a new value upon the application of TMS $\left(\lambda_{T M S}\right)$. Pilot studies were performed to estimate approximate parameter ranges (based on minimizing error between experimental and simulated results), after which the $\sigma$ parameter was allowed to vary freely within the range $(1.00,3.00)$, with a stepsize of $0.5 ; \lambda_{\text {baseline }}$ was fixed at 0.5 , and $\lambda_{T M S \text { (ipsilateral) }}$ and $\lambda_{T M S \text { (contralateral) were varied across }}$ the range of $(0.5,0.025)$, taking possible values of $0.5,0.375$, $0.25,0.125,0.1,0.075,0.05$, and 0.025 . Two thousand trials were simulated for each combination of onset time and free parameter values. Parameter fit was assessed by minimizing the sum of squared error (SS) between the experimental results from Harris et al. (2002) (rounded to four places) and simulated results, and the selected parameter values were those that minimized total SS across both ipsilateral and contralateral TMS conditions. (Note that parameter selection was constrained by requiring the value of $\sigma$ to be the same for both ipsilateral and contralateral stimulation conditions).

To improve the model fit, a second round of simulations was performed based on the best-fitting parameters from the first round of simulations $\left[\sigma=2.00, \lambda_{T M S}\right.$ (ipsilateral) $=0.375$, and $\left.\lambda_{T M S \text { (contralateral })}=0.125\right]$. The value of $\sigma$ was set to 2.00 , and $\lambda_{\text {TMS(ipsilateral) }}$ and $\lambda_{\text {TMS(contralateral) }}$ varied within the ranges $(0.425,0.325)$ and $(0.175,0.075)$, respectively, with a stepsize of 0.025 .

Simulations were performed with code written in Python, with the NumPy and standard Python random libraries (specifically, random.expovariate for the generation of noisy input). Integration was performed using a 4th-order Runge-Kutta, with an integration stepsize of 0.5 .

\section{RESULTS AND DISCUSSION}

The results of the final round of simulations are presented in Figures 2, 3. The best-fitting parameter values were found to be $\sigma=2.0, \lambda_{T M S \text { (ipsilateral })}=0.350$, and $\lambda_{T M S(\text { contralateral })}=0.150$. The SS for the best fit was found to be $0.00446(0.00273$ for the ipsilateral condition, and 0.00173 for the contralateral condition), and the variance explained by the model $\left(r^{2}\right)$ was calculated to be 0.780 .

Model performance was largely robust against changes in parameter values, with maximum overall $S S$ of 0.0962 in the final round of simulations $(0.00485$ for the ipsilateral condition, and 0.0914 for the contralateral condition).

The results of the present simulation suggest that Harris et al.'s (2002) results can be replicated by assuming that TMS increases activity in sensory cortex, which then interferes with the contents of memory, held in PFC. This interpretation is consistent with the
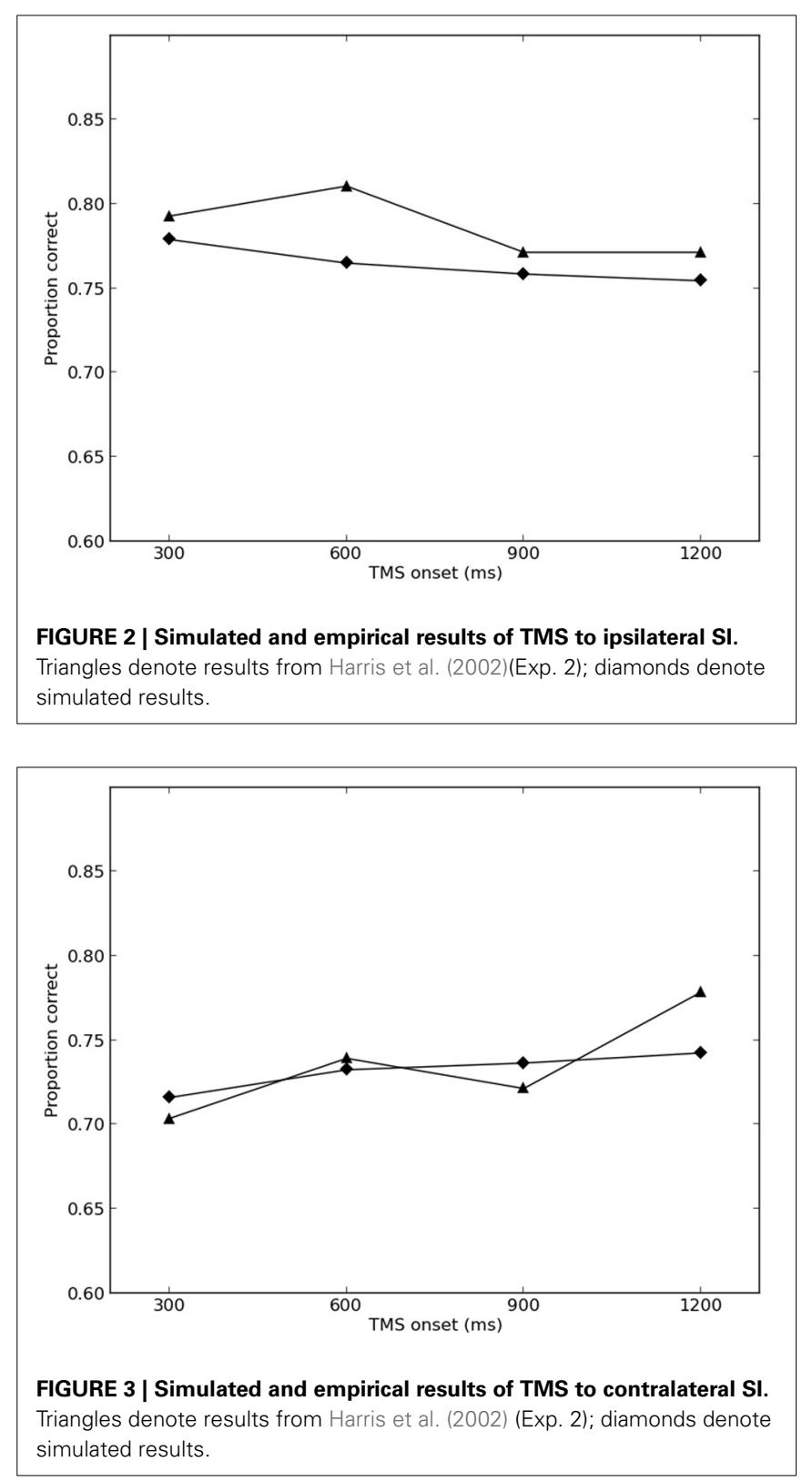

single-cell electrophysiology and EEG literatures (e.g., Romo and Salinas, 2003; Spitzer et al., 2010, 2014; Spitzer and Blankenburg, 2011, 2012), and requires no need to suggest that SI is involved in vibrotactile memory storage.

One crucial part of Harris et al.'s argument was that TMS to SI ipsilateral to the hand receiving vibrotactile stimulation did not produce effects on task performance. They suggested that if VWM storage relied (at least in part) on areas further downstream, such as SII (which possesses bilateral receptive fields), TMS to ipsilateral cortex would produce similar effects to TMS to contralateral cortex. However, recent EEG and MEG studies of tactile memory have reported greater alpha-band activity over ipsilateral SI than over contralateral SI (Haegens et al., 2010, 2012; Spitzer and Blankenburg, 2011). Further, when irrelevant stimuli were expected to be presented to the opposite hand, pre-stimulus 
alpha power in cortex varied with laterality (Haegens et al., 2012). As alpha-band activity is believed to be linked to inhibitory activity (e.g., Rihs et al., 2007; Haegens et al., 2011), Haegens et al. (2012) suggested that activity in ipsilateral SI could be suppressed in order to inhibit the processing of irrelevant sensory input. In this case, the failure to find effects of ipsilateral TMS does not necessarily reflect a reliance on contralateral SI for VWM storage, but rather may reflect differences in endogenous inhibitory activity between ipsilateral and contralateral sensory cortex.

The results of the present study have an impact reaching beyond the VWM literature. Postle (2006) introduced the emergent-property model of working memory, which suggests that working memory does not rely on a specialized neural system, but rather the interaction between neural systems that primarily serve other sensory, cognitive, or action-related functions. Indeed, Postle explicitly argues that PFC is not involved in the storage of information. For example, task-relevant sensory cortex has been suggested as the storage medium for working memory, and recent neuroimaging studies that have applied novel methods for decoding the contents of sensory cortex have reported finding stimulus information in early visual cortex during the maintenance period of visual memory tasks (e.g., Serences et al., 2009; Christophel et al., 2012). Other, similar results exist.

However, there is an increasing body of evidence that PFC is the storage substrate for simple stimuli and novel stimuli (e.g., Freedman et al., 2001; Bancroft et al., 2014). Perhaps most persuasive are recent studies that have reported decoding the contents of short-term or working memory from prefrontal beta-band activity, regardless of whether the stored aspect of the stimulus was delivered as a tactile vibration, auditory tone, visual flicker (Spitzer et al., 2010; Spitzer and Blankenburg, 2012), or stimulus intensity or duration (Spitzer et al., 2014). While the emergentproperty model is compelling, in that it is simple, parsimonious and able to explain a wide variety of results from the literature, when combined with previous findings, the results of the present study suggest that VWM research may require an expansion of the emergent-property model. We have recently suggested that the complexity of a stimulus is at least a partial factor in determining what neural storage systems are recruited (Bancroft et al., 2014).

We acknowledge that the timecourse of the effects of TMS to SI are not well-understood. Indeed, the effects of TMS to SI are not well-understood in general. Harris et al. (2002) selected a target in SI by using TMS to identify the region of greatest tactile extinction, which could be interpreted as evidence of an inhibitory, rather than excitatory effect of TMS. However, other research has found excitatory or facilitatory effects of TMS over sensory and motor cortex (Gerwig et al., 2003; Ragert et al., 2004, 2008), and even combined excitatory and inhibitory effects (Oliveri et al., 2000; Moliadze et al., 2003; Strafella et al., 2004). Indeed, even inhibitory effects on neurons in a stimulated region can produce increased neural activity or excitability, due to a reduction in the activity of inhibitory interneurons. Further, Amassian et al. (2002) suggest that single-pulse TMS can excite a large number of neurons in sensory cortex without the effects reaching consciousness.
Effects are also likely to depend heavily on cortical structure and connectivity. Identical stimulation parameters can produce excitation or inhibition in different cortical regions (Paus, 2005), and there is growing evidence that the effects of TMS over a cortical region are state-dependent, with effects possibly depending on pre-existing activity in the region (Harris et al., 2008; Pasley et al., 2009; Abrahamyan et al., 2011). Recently, a number of authors (including Harris) have suggested that TMS resulting in what appears to be inhibitory behavioral effects can actually be due to increased neural excitability resulting in an unfavorable signalto-noise ratio (Silvanto and Muggleton, 2008; Miniussi et al., 2013).

Whether TMS-induced behavioral results are driven by cortical inhibition, an unfavorable neuronal signal-to-noise ratio, or both to some extent, the present work highlights another critical issue: the local vs. remote interpretation of the neural intervention. Combined TMS/fMRI studies have shown that, even at relatively low intensities, TMS modulates hemodynamic activity in both the targeted brain region and distant cortical and subcortical regions (e.g., Bohning et al., 1999; Bestmann et al., 2005). Though the distinction between local or remote effects of TMS may be inconsequential in some settings, in the case of Harris et al.'s (2002) findings, interpreting the effects of TMS as related to SI inhibition or PFC signal-to-noise modulation produce fundamentally different insights for neurocognitive models of STM storage. In such a case, it might be useful to, wherever possible, choose multiple stimulation sites (e.g., SI and PFC) and timings (e.g., early vs. late in the delay period at both sites) in order to design experiments that use TMS to conclusively elucidate the where and when of a given cognitive task in the brain.

It is likely that relatively limited activity in SI can produce effects downstream, given the feedforward nature of output connections from SI (Romo and Salinas, 2003). When discussing Harris et al.'s (2002) results, Romo and Salinas (2003) suggested that TMS was likely to produce localized effects in cortex for approximately $200 \mathrm{~ms}$ after application. This may be a conservative estimate; others have reported that the initial phase of increased neural activity can persist for approximately $500 \mathrm{~ms}$ after TMS application (Moliadze et al., 2003; Silvanto and Muggleton, 2008). However, given that the effects of activity in SII produced by vibrotactile stimuli can persist for several hundred milliseconds after stimulus offset (Romo and Salinas, 2003), as well as possible feedback loops within the vibrotactile memory system (e.g., Auksztulewicz et al., 2012), it appears plausible that effects of a TMS pulse in SI could produce longer-lasting effects downstream, and could produce substantial interference with VWM storage. Experimental tests of this hypothesis (possibly involving a combined TMS/ERP paradigm) should prove fruitful.

In the present study, we have suggested a way to integrate the TMS results of Harris et al. (2002) with the EEG and singlecell literatures. The present study also poses a challenge to the emergent-property model of working memory, and suggests a manner in which that model may be extended. Finally, and perhaps most intriguingly, the results of the current study are consistent with growing evidence that short-term and working memory may rely on different neural storage substrates, based on 
the salient property of the stimulus that is being maintained in memory; for example, in the present study, we have shown that simple tactile stimuli are stored in PFC (as are simple stimuli in other sensory modalities, see Bancroft et al., 2014) but complex tactile stimuli may be stored in sensory cortex (e.g., Zhou and Fuster, 1996).

\section{ACKNOWLEDGMENTS}

We are indebted to Justin Harris for providing us with the means from Experiment 2 of Harris et al. (2002), as well as for granting permission to publish that data. We thank Britt Anderson, Diano Marrone, and the reviewers for their helpful comments. This research was supported by funding from the Wilfrid Laurier University Library, grants from the Natural Science and Engineering Research Council to William E. Hockley and Philip Servos, an Ontario Graduate Scholarship to Tyler D. Bancroft, and a Social Science and Humanities Research Council graduate scholarship to Jeremy Hogeveen.

\section{REFERENCES}

Abrahamyan, A., Clifford, C. W. G., Arabzadeh, E., and Harris, J. A. (2011). Improving visual sensitivity with subthreshold transcranial magnetic stimulation. J. Neurosci. 31, 3290-3294. doi: 10.1523/JNEUROSCI.625610.2011

Amassian, V. E., Cracco, R. Q., Maccabee, P. J., and Cracco, J. B. (2002). "Visual system," in Handbook of Transcranial Magnetic Stimulation, eds A. Pascual-Leone, N. J. Davey, J. Rothwell, E. M. Wasserman, and B. K. Puri (London: Arnold), 323-334.

Auksztulewicz, R., Spitzer, B., and Blankenburg, F. (2012). Recurrent neural processing and somatosensory awareness. J. Neurosci. 32, 799-805. doi: 10.1523/JNEUROSCI.3974-11.2012

Bancroft, T. D., Hockley, W. E., and Servos, P. (2011a). Vibrotactile working memory as a model paradigm for psychology, neuroscience, and computational modeling. Front. Hum. Neurosci. 5:162. doi: 10.3389/fnhum.2011.00162

Bancroft, T. D., Hockley, W. E., and Servos, P. (2012). Diffusion modeling of interference in vibrotactile working memory. Neuroreport 23, 255-258. doi: 10.1097/WNR.0b013e3283507550

Bancroft, T. D., Hockley, W. E., and Servos, P. (2013). Irrelevant sensory stimuli interfere with working memory storage: evidence from a computational model of prefrontal neurons. Cogn. Affect. Behav. Neurosci. 13, 23-34. doi: 10.3758/s13415-012-0131-9

Bancroft, T. D., Hockley, W. E., and Servos, P. (2014). Does stimulus complexity determine whether working and short-term memory storage relies on prefrontal cortex or sensory cortex? Attent. Percept. Psychophys. doi: 10.3758/s13414-0130604-0. [Epub ahead of print].

Bancroft, T. D., Servos, P., and Hockley, W. E. (2011b). Mechanisms of interference in vibrotactile working memory. PLOS ONE 6:e22518. doi: 10.1371/journal.pone.0022518

Bancroft, T., and Servos, P. (2011). Distractor frequency influences performance in vibrotactile working memory. Exp. Brain Res. 208, 529-532. doi: 10.1007/s00221-010-2501-2

Bestmann, S., Baudewig, J., Siebner, H. R., Rothwell, J. C., and Frahm, J. (2005). BOLD MRI responses to repetitive TMS over human dorsal premotor cortex. Neuroimage 28, 22-29. doi: 10.1016/j.neuroimage.2005.05.027

Bohning, D. E., Shastri, A., McConnell, K. A., Nahas, Z., Lorberbaum, J. P., Roberts, D. R., et al. (1999). A combined TMS/fMRI study of intensity-dependent TMS over motor cortex. Biol. Psychiatry 45, 385-394. doi: 10.1016/S00063223(98)00368-0

Christophel, T. B., Hebard, M. N., and Haynes, J. (2012). Decoding the contents of visual short-term memory from human visual and parietal cortex. J. Neurosci. 32, 12983-12989. doi: 10.1523/JNEUROSCI.0184-12.2012

Freedman, D. J., Riesenhuber, M., Poggio, T., and Miller, E. K. (2001). Categorical representation of visual stimuli in the primate prefrontal cortex. Science 291, 312-316. doi: 10.1126/science.291.5502.312

Gerwig, M., Kastrup, O., Meyer, B.-U., and Niehaus, L. (2003). Evaluation of cortical excitability by motor and phosphene thresholds in transcranial magnetic stimulation. J. Neurol. Sci. 215, 75-78. doi: 10.1016/S0022-510X (03)00228-4

Haegens, S., Luther, L., and Jensen, O. (2012). Somatosensory anticipatory alpha activity increases to suppress distracting input. J. Cogn. Neurosci. 24, 677-685. doi: 10.1162/jocn_a_00164

Haegens, S., Nácher, V., Luna, R., Romo, R., and Jensen, O. (2011). $\alpha$-Oscillations in the monkey sensorimotor network influence discrimination performance by rhythmical inhibition of neuronal spiking. Proc. Natl. Acad. Sci. U.S.A. 108, 19377-19382. doi: 10.1073/pnas.1117190108

Haegens, S., Osipova, D., Oostenveld, R., and Jensen, O. (2010). Somatosensory working memory performance in humans depends on both engagement and disengagement of regions in a distributed network. Hum. Brain Mapp. 31, 26-35. doi: 10.1002/hbm.20842

Hannula, H., Neuvonen, T., Savolainen, P., Hiltunen, J., Ma, Y., Antila, H., et al. (2010). Increasing top-down suppression from prefrontal cortex facilitates tactile working memory. Neuroimage 49, 1091-1098. doi: 10.1016/j.neuroimage.2009.07.049

Harris, J. A., Clifford, C. W., and Miniussi, C. (2008). The functional effect of transcranial magnetic stimulation: signal suppression or neural noise generation. J. Cogn. Neurosci. 20, 734-740. doi: 10.1162/jocn.2008.20048

Harris, J. A., Miniussi, C., Harris, I. M., and Diamond, M. E. (2002). Transient storage of a tactile memory trace in primary somatosensory cortex. J. Neurosci. 22, 8720-8725.

Miller, P., and Wang, X.-J. (2006). Inhibitory control by an integral feedback signal in prefrontal cortex: a model of discrimination between sequential stimuli. Proc. Natl. Acad. Sci. U.S.A. 103, 201-206. doi: 10.1073/pnas.0508072103

Miniussi, C., Harris, J. A., and Ruzzoli, M. (2013). Modelling non-invasive brain stimulation in cognitive neuroscience. Neurosci. Biobehav. Rev. 37, 1702-1712. doi: 10.1016/j.neubiorev.2013.06.014

Moliadze, V., Zhao, Y., Eysel, U., and Funke, K. (2003). Effect of transcranial magnetic stimulation on single-unit activity in the cat primary visual cortex. J. Physiol. 553, 665-679. doi: 10.1113/jphysiol.2003.050153

Oliveri, M., Caltagirone, C., Filippi, M. M., Traversa, R., Cicinelli, P., Pasqualetti, P., et al. (2000). Paired transcranial magnetic stimulation protocols reveal a pattern of inhibition of facilitation in the human parietal cortex. J. Physiol. 529, 461-468. doi: 10.1111/j.1469-7793.2000.00461.x

Pasley, B. N., Allen, E. A., and Freeman, R. D. (2009). State-dependent variability of neuronal responses to transcranial magnetic stimulation of the visual cortex. Neuron 62, 291-303. doi: 10.1016/j.neuron.2009.03.012

Paus, T. (2005). Inferring causality in brain images: a perturbation approach. Philos. Trans. R. Soc. B Biol. Sci. 360, 1109-1114. doi: 10.1098/rstb.2005.1652

Paus, T., Jech, R., Thompson, C. J., Comeau, R., Peters, T., and Evans, A. C. (1997). Transcranial magnetic stimulation during positron emission tomography: a new method for studying connectivity of the human cerebral cortex. J. Neurosci. 17, 3178-3184.

Postle, B. R. (2006). Working memory as an emergent property of the mind and brain. Neuroscience 139, 23-38. doi: 10.1016/j.neuroscience.2005.06.005

Ptito, M., Fumal, A., Martens de Noordhout, A., Schonen, J., Gjedde, A., and Kupers, R. (2008). TMS of the occipital cortex induces tactile sensations in the fingers of blind Braille readers. Exp. Brain Res. 184, 193-200. doi: 10.1007/s00221-007-1091-0

Ragert, P., Becker, M., Tegenthoff, M., Pleger, B., and Dinse, H. R. (2004). Sustained increase of somatosensory cortex excitability by $5 \mathrm{~Hz}$ repetitive transcranial magnetic stimulation studied by paired median nerve stimulation in humans. Neurosci. Lett. 356, 91-94. doi: 10.1016/j.neulet.2003.11.034

Ragert, P., Franzkowiak, S., Schwenkreis, P., Tegenthoff, M., and Dinse, H. R. (2008). Improvement of tactile perception and enhancement of cortical excitability through intermittent theta burst rTMS over human primary somatosensory cortex. Exp. Brain Res. 184, 1-11. doi: 10.1007/s00221-0071073-2

Ray, P. G., Meador, K. J., Epstein, C. M., Loring, D. W., and Day, L. J. (1998). Magnetic stimulation of visual cortex: factors influencing the perception of phosphenes. J. Clin. Neurophysiol. 15, 351-357. doi: 10.1097/00004691199807000-00007

Rihs, T. A., Michel, C. M., and Thut, G. (2007). Mechanisms of selective inhibition in visual spatial attention are indexed by $\alpha$-band EEG synchronization. Eur. J. Neurosci. 25, 603-610. doi: 10.1111/j.1460-9568.2007.05278.x

Robertson, E. M., Théoret, H., and Pascual-Leone, A. (2003). Studies in cognition: the problems solved and created by transcranial magnetic stimulation. J. Cogn. Neurosci. 15, 948-960. doi: 10.1162/089892903770007344 
Romo, R., and Salinas, E. (2003). Flutter discrimination: Neural codes, perception, memory and decision making. Nat. Rev. Neurosci. 4, 203-218. doi: 10.1038/nrn1058

Romo, R., Brody, C. D., Hernández, A., and Lemus, L. (1999). Neuronal correlates of parametric working memory in the prefrontal cortex. Nature 399, 470-473. doi: $10.1038 / 20939$

Romo, R., Hernández, A., Zainos, A., and Salinas, E. (1998). Somatosensory discrimination based on cortical microstimulation. Nature 392, 387-390. doi: $10.1038 / 32891$

Savolainen, P., Carlson, S., Boldt, R., Neuvonen, T., Hannula, H., Hiltunen, J., et al. (2011). Facilitation of tactile working memory by top-down suppression from prefrontal to primary somatosensory cortex during sensory interference. Behav. Brain Res. 219, 387-390. doi: 10.1016/j.bbr.2011.01.053

Serences, J. T., Ester, E. F., Vogel, E. K., and Awh, E. (2009). Stimulus-specific delay activity in human primary visual cortex. Psychol. Sci. 20, 207-214. doi: 10.1111/j.1467-9280.2009.02276.x

Silvanto, J., and Muggleton, N. G. (2008). New light through old windows: moving beyond the "virtual lesion" approach to transcranial magnetic stimulation. Neuroimage 39, 549-552. doi: 10.1016/j.neuroimage.2007.09.008

Spitzer, B., and Blankenburg, F. (2011). Stimulus-dependent EEG activity reflects internal updating of tactile working memory in humans. Proc. Natl. Acad. Sci.U.S.A. 108, 8444-8449. doi: 10.1073/pnas.1104189108

Spitzer, B., and Blankenburg, F. (2012). Supramodal parametric working memory processing in humans. J. Neurosci. 32, 3287-3295. doi: 10.1523/JNEUROSCI.5280-11.2012

Spitzer, B., Gloel, M., Schmidt, T. T., and Blankenburg, F. (2014). Working memory coding of analog stimulus properties in the human prefrontal cortex. Cereb. Cortex. [Epub ahead of print].

Spitzer, B., Wacker, E., and Blankenburg, F. (2010). Oscillatory correlates of vibrotactile frequency processing in human working memory. J. Neurosci. 30, 4496-4502. doi: 10.1523/JNEUROSCI.6041-09.2010
Stewart, L. M., Walsh, V., and Rothwell, J. C. (2001). Motor and phosphene thresholds: a transcranial magnetic stimulation correlation study. Neuropsychologia 39, 415-419. doi: 10.1016/S0028-3932 (00)00130-5

Strafella, A. P., Vanderwerf, Y., and Sadikot, A. F. (2004). Transcranial magnetic stimulation of the human motor cortex influences the neuronal activity of subthalamic nucleus. Eur. J. Neurosci. 20, 2245-2249. doi: 10.1111/j.14609568.2004.03669.x

Sugishita, M., and Takayama, Y. (1993). Paraesthesia elicited by repetitive magnetic stimulation of the postcentral gyrus. Neuroreport 4, 569-570. doi: 10.1097/00001756-199305000-00027

Zhou, Y. D., and Fuster, J. M. (1996). Mnemonic neuronal activity in somatosensory cortex. Proc. Natl. Acad. Sci. U.S.A. 93, 10533-10537. doi: 10.1073/pnas.93.19.10533

Conflict of Interest Statement: The authors declare that the research was conducted in the absence of any commercial or financial relationships that could be construed as a potential conflict of interest.

Received: 15 July 2013; accepted: 10 February 2014; published online: 05 March 2014. Citation: Bancroft TD, Hogeveen J, Hockley WE and Servos P (2014) TMS-induced neural noise in sensory cortex interferes with short-term memory storage in prefrontal cortex. Front. Comput. Neurosci. 8:23. doi: 10.3389/fncom.2014.00023

This article was submitted to the journal Frontiers in Computational Neuroscience. Copyright (c) 2014 Bancroft, Hogeveen, Hockley and Servos. This is an openaccess article distributed under the terms of the Creative Commons Attribution License (CC BY). The use, distribution or reproduction in other forums is permitted, provided the original author(s) or licensor are credited and that the original publication in this journal is cited, in accordance with accepted academic practice. No use, distribution or reproduction is permitted which does not comply with these terms. 